\title{
Non-Coherent and Semi-Coherent Schemes for Physical-Layer Wireless Network Coding
}

\author{
Zoran Utkovski* and Petar Popovski ${ }^{\dagger}$ \\ ${ }^{*}$ Faculty of Informatics, University Goce Delcev Stip, Republic of Macedonia \\ $\dagger$ Department of Electronic Systems, Aalborg University, Denmark
}

\begin{abstract}
We investigate non-coherent and semi-coherent schemes for physical-layer network coding in two-way relaying scenarios. We distinguish between scenarios without any channel knowledge requirements (non-coherent communication) and scenarios when either the relay or the users have receive channel knowledge (semi-coherent communication). We combine the paradigm of subspace-based communication originally developed for non-coherent point-to-point channels, with two-way relaying schemes based on physical-layer wireless network coding with denoise-and- forward (DNF). The aim is to demonstrate that denoising can be performed non-coherently and to investigate if these schemes offer an improvement over the schemes based on amplify-and-forward (AF).
\end{abstract}

\section{INTRODUCTION}

Most schemes for relaying require prior knowledge of the channel at the terminals and at the relay(s). Although sometimes taken for granted, the assumption of perfect channel knowledge is not always realistic and depends heavily on the system model. For example, in the case of quasi-static fading the channel remains constant (or changes slowly) over a longer period of time, typically over hundreds or thousands of symbols. On the other hand, the widely used block fading model introduced by Hochwald and Marzetta [1] assumes that the channel is constant in a given time block and then changes in an independent realization. If this block length is short, for example only several symbols, it might be difficult to estimate the channel. Even the differential schemes used for quasi-static channels might not be applicable in this setup, since they have to be re-initialized in every time block.

With this discussion on mind, we will investigate schemes for wireless network coding in two-way relaying scenarios with different degrees of channel knowledge, all of practical relevance. In the first scenario we focus on two-way relaying without any channel knowledge requirements at the terminals and at the relays. This scenario is of interest when it is difficult for both the terminals and the relay to estimate the channel coefficients and therefore the communication schemes should be non-coherent. The second scenario involves partial channel knowledge at the receivers and therefore the scheme is referred to as semi-coherent. This scenario assumes channel knowledge at the terminals (link relay-terminal), but not at the relay and is of interest when, for example, the relay is constrained to do relatively simple operations which do not involve sophisticated channel estimation techniques.

\section{SySTEM MODEL}

We consider a wireless network with two users, A and $\mathrm{B}$, one relay node $\mathrm{R}$ and no direct link between the terminals. All the transceivers (terminals and relay) work in a half-duplex regime i. e. they can not transmit and receive simultaneously. We assume block Rayleigh model where the channel is constant in a certain time block. The communication takes part in two phases. The first phase is the multiple access (MA) phase, where both users simultaneously transmit their information. The signals transmitted from the users are combined at the relay $\mathrm{R}$, which performs a certain operation on the received signal, depending on the relaying strategy. In the next phase, denoted as broadcast phase (BC) the relay $\mathrm{R}$ broadcasts a signal to both users. Based on the received signal and the knowledge about its' own transmitted signal, each user decodes the information from the other user.

The signal transmitted from user $\mathrm{A}$ is a $T \times 1$ vector $\sqrt{P T} \mathbf{x}_{A}$, normalized such that $\mathrm{E}\left[\operatorname{tr}\left(\mathbf{x}_{A}^{\mathrm{H}} \mathbf{x}_{A}\right)\right]=1$. The codebook of user $\mathrm{A}$ is denoted as $\mathcal{X}_{A}$. Similarly, user $\mathrm{B}$ sends a $T \times 1$ transmit vector $\sqrt{P T} \mathbf{x}_{B}$, normalized such that $\mathrm{E}\left[\operatorname{tr}\left(\mathbf{x}_{B}^{\mathrm{H}} \mathbf{x}_{B}\right)\right]=1$. The codebook of user $\mathrm{B}$ is denoted as $\mathcal{X}_{B}$. $P$ is the average transmit power for one transmission of user $A$ and user B. Further, we denote the average power for one transmission for the relay as $P_{R}$. Additionally, we have the constraint on the total network power, $2 P+P_{R}=P_{t o t}$ which serves for fair comparison, since it considers the transmit powers of all network nodes.

Within one block $T$, the channel between A and R (MA phase) is denoted as $h^{\prime}$ and the channel between $\mathrm{R}$ and $\mathrm{A}$ in the BC phase as $h$ ". If not explicitly mentioned, we will assume that these channel realizations are different and independent. Similarly, the channel between B and R in the MA phase is denoted as $g^{\prime}$ and the in the BC phase as $g$ ". Hence, after the MA phase, the relay $R$ receives the signal

$$
\mathbf{y}_{R}=\sqrt{P T} \mathbf{x}_{A} h^{\prime}+\sqrt{P T} \mathbf{x}_{B} g^{\prime}+\mathbf{z}_{R},
$$

which can be written in the form

$$
\mathbf{y}_{R}=\sqrt{P T}\left[\begin{array}{ll}
\mathbf{x}_{A} & \mathbf{x}_{B}
\end{array}\right]\left[\begin{array}{c}
h^{\prime} \\
g^{\prime}
\end{array}\right]+\mathbf{z}_{R}
$$

where $\mathbf{z}_{R}$ is the noise vector at the relay $\mathrm{R}$, with elements which are i.i.d. complex Gaussian, $C N(0,1)$. From the point of view of the relay $\mathrm{R}$, we can think of $\left[\begin{array}{ll}\mathbf{x}_{A} & \mathbf{x}_{B}\end{array}\right]$ as an equivalent tranmit matrix and of $\left[\begin{array}{c}h^{\prime} \\ g^{\prime}\end{array}\right]$ as an equivalent 
channel. This system model will be used for the investigation of the scenarios of interest, addressed in the rest of the paper.

\section{RELAY AND TERMINALS WITHOUT CHANNEL KNOWLEDGE}

This scenario has been partly investigated in [8], [2], [3], where communication schemes based on amplify-and-forward (AF) were presented. The scheme proposed in [8] is a differential scheme, generalizing differential transmission from oneway to two-way relaying. The scheme proposed in [3] is based on subspace communication in the spirit of the geometric approach introduced in [5]. This scheme is motivated by the non-coherent schemes designed for point-to-point channels and relies on the interpretation of the codebook design as a sphere packing problem in Grassmann manifolds. Let us look for ilustration at the system model for terminals equipped with single antenna, where the received signal is given as:

$$
\mathbf{y}=\mathbf{x} h+\mathbf{w} .
$$

Since the channel $h$ acts on the transmit vector $\mathbf{x}$ only as a scaling factor, the 1-dimensional subspace of the complex space $\mathbb{C}^{T}$ spanned by $\mathbf{x}$ does not change after the multiplication by the channel coefficient. In other words, we can send information over the unknown channel by simply sending information about a subspace of the complex space $\mathbb{C}^{T}$ spanned by $\mathbf{x}$. This naturally defines our space of transmit signals, or our coding space, to be the set of all 1-dimensional linear subspaces of $\mathbb{C}^{T}$. This set has a structure of a manifold and is known as the Grassmann manifold $G_{1, T}^{\mathbb{C}}$. We note that the generalization to MIMO systems is straightforward. For system with $M$ transmit antennas, the information is carried by the $M$-dimensional subspace spanned by the columns of the transmit matrix $\mathbf{X}$, instead of the transmit vector $\mathbf{x}$.

We will address two schemes, amplify-and-forward (AF) and denoise-and-forward (DNF) and compare them in the noncoherent setup.

\section{A. Amplify-and-forward}

We start by observing the signal $\mathbf{y}_{R}$ received at the relay, according to the system model (9). This is similar to the system model for the non-coherent MIMO point-to-point block fading channel with 2 transmit, 1 receive antennas and coherence time $T$. We should note that $T \geq 4$ is required in this context [5]. Let us denote by $\mathbf{X}_{A B}$ the matrix obtained by the concatenation of $\mathbf{x}_{A}$ and $\mathbf{x}_{B} \mathbf{X}_{A B}=\left[\begin{array}{ll}\mathbf{x}_{A} & \mathbf{x}_{B}\end{array}\right]$. The columns of $\mathbf{X}_{A B}$ span a 2-dimensional subspace, which can be represented by the orthonormal basis $\mathbf{Q}$, where $\mathbf{X}_{A B}=\mathbf{Q R}$ is the $\mathrm{QR}$ decomposition. In order to distinguish between the matrix $Q$ and the subspace spanned by the columns of the matrix, we denote the subspace as $\Omega_{\mathrm{Q}}$. Howewer, for simplicity of the notation, we will sometimes adhere to $\mathbf{Q}$ as both the matrix and the subspace spanned by the columns of the matrix. Additionally, we denote by $\mathcal{Q}$ the set of the subspaces obtained by the above concatenation. The cardinality of the codebook is at most $|\mathcal{Q}|=\left|\mathcal{X}_{A}\right|\left|\mathcal{X}_{B}\right|$.
In [3] it has been shown that by using AF strategy at the relay it is possible for the terminals to decode the received signal. Indeed, with AF, the relay $\mathrm{R}$ broadcasts the signal $\mathbf{x}_{R}=$ $\sqrt{\frac{P_{R}}{1+2 P}} \mathbf{r}$. The received signal at user $\mathrm{B}$ is in the form

$$
\mathbf{y}_{B}=\sqrt{\frac{P P_{R} T}{1+2 P}}\left[\begin{array}{ll}
\mathbf{x}_{A} & \mathbf{x}_{B}
\end{array}\right]\left[\begin{array}{l}
h^{\prime} h^{\prime \prime} \\
g^{\prime} h^{\prime \prime}
\end{array}\right]+\mathbf{w}_{B},
$$

where $\mathbf{w}_{B}$ is the equivalent noise at user $\mathrm{B}$, having contribution from the relay noise as well,

$$
\mathbf{w}_{B}=\sqrt{\frac{P_{R}}{1+2 P}} \mathbf{z}_{R}+\mathbf{z}_{B} .
$$

We can perform the decoding by looking for the most likely transmitted subspace from the set of subspaces $\mathcal{Q}$, having the received vector $\mathbf{y}_{B}$

$$
\hat{\mathbf{Q}}=\arg \max _{\mathbf{Q}_{i} \in \mathcal{Q}}\left\|\mathbf{y}_{B}^{\mathrm{H}} \mathbf{Q}_{i}\right\|_{F}^{2}
$$

Having the subspace $\mathbf{Q}$, we get the pair $\left(\mathbf{x}_{A}, \mathbf{x}_{B}\right)$. When looking for the most likely subspace $\mathbf{Q}$ we can use the fact that we know $\mathbf{x}_{B}$ in advance, which limits the number of the subspaces we have to search.

\section{B. Denoise-and-forward}

Preliminary investigations [2] show that AF achieves the degrees of freedom in the limit of large number of relays and high SNR. However, schemes based on the denoise-andforward (DNF) technique introduced in [6] and used in [7], might outperform AF in the case of one relay or finite (small) number of relays, as well as limited SNR. In this context, we will investigate the possibility of using DNF schemes in the non-coherent setup.

The basic principle behind DNF is that in the MA stage, the relay $\mathrm{R}$ does not jointly decode the signals from $\mathrm{A}$ and $\mathrm{B}$, but it maps the received signals into symbols from a discrete constellation and broadcasts. The simplest scenario to illustrate the basic idea of DNF is when the terminals use BPSK modulation in the MA stage. Assume that the channel gains are 1 and the transmitted signals are $x_{A}, x_{B} \in\{1,-1\}$. The signal received by the relay is $y_{R}=x_{A}+x_{B}+z_{R}$. If the channel in the MA stage are noiseless $\left(z_{R}=0\right)$, then the possible received signals at the relay $\mathrm{R}$ are $\{-2,0,2\}$. Clearly, if $y_{R}=0, \mathrm{R}$ has residual ambiguity and cannot jointly decode the signals from $\mathrm{A}$ and $\mathrm{B}$ even in a noiseless case. Nevertheless, $\mathrm{R}$ can use the following denoising map: If it receives -2 or 2 , it sends -1 in the $\mathrm{BC}$ stage, while if it receives 0 , it sends 1 in the $\mathrm{BC}$ stage. Now, if $\mathrm{A}$ receives -1 from $\mathrm{R}$ and it knows a priori that it has sent -1 during the MA stage, then it interprets that B has sent the symbol -1 .

In a more formal description, the relay $\mathrm{R}$ employs a denoising function to map the received signal $y_{R}$ into a quantized signal $x_{R}$ for the BC stage. The denoising function consists of a denoising mapper $\mathcal{C}$ and a constellation mapper $\mathcal{M}_{R}$, preceded by the maximumlikelihood (ML) joint detection. The denoising map $\mathcal{C}$ generates a networkcoded data from the ML 
estimates. This data is then broadcasted after being modulated with the relay constellation mapper $\mathcal{M}_{R}$.

Yet, the denoise-and forward scheme in its' original form requires channel knowledge at the relay. We will attempt to design a denoise-and-forward scheme which does not require channel knowledge. We recall that the communication in the non-coherent setup is based on transmitting information about subspaces, rather then transmitting symbols from some alphabet. In this context, the codebooks of the users $A$ and $\mathrm{B}, \mathcal{X}_{A}$ and $\mathcal{X}_{B}$, represent sets of subspaces. The problem of finding appropriate DNF schemes can be formulated in the following way. Based on the subspace spanned by the received vector $\mathbf{y}_{R}$, the relay has to find a denoising map $\mathcal{C}$ without jointly decoding the subspaces transmitted by $\mathrm{A}$ and $\mathrm{B}$. In the following we propose a communication scheme which operates non-coherently and is combined with denoiseand-forward at the relay. We will describe the communication protocol in details, by looking separately at all phases of the transmission.

1) MA Stage: We choose the codebooks of user A and user $\mathrm{B}$ as follows

$\mathcal{X}_{A}=\sin \alpha\left\{\left[\begin{array}{c}\cot \alpha \\ 0 \\ \frac{(1+i)}{\sqrt{2}} \\ \frac{(1-i)}{\sqrt{2}}\end{array}\right],\left[\begin{array}{c}\cot \alpha \\ 0 \\ \frac{1-i}{\sqrt{2}} \\ \frac{1+i}{\sqrt{2}}\end{array}\right],\left[\begin{array}{c}\cot \alpha \\ 0 \\ \frac{-1-i}{\sqrt{2}} \\ \frac{-1+i}{\sqrt{2}}\end{array}\right],\left[\begin{array}{c}\cot \alpha \\ 0 \\ \frac{-1+i}{\sqrt{2}} \\ \frac{-1-i}{\sqrt{2}}\end{array}\right]\right\}$
$\mathcal{X}_{B}=\sin \alpha\left\{\left[\begin{array}{c}0 \\ \cot \alpha \\ \frac{-1+i}{\sqrt{2}} \\ \frac{1+i}{\sqrt{2}}\end{array}\right],\left[\begin{array}{c}0 \\ \cot \alpha \\ \frac{1+i}{\sqrt{2}} \\ \frac{-1+i}{\sqrt{2}}\end{array}\right],\left[\begin{array}{c}0 \\ \cot \alpha \\ \frac{1-i}{\sqrt{2}} \\ \frac{-1-i}{\sqrt{2}}\end{array}\right],\left[\begin{array}{c}0 \\ \cot \alpha \\ \frac{-1-i}{\sqrt{2}} \\ \frac{1-i}{\sqrt{2}}\end{array}\right]\right\}$

where for the parameter $\alpha$ we take $\alpha=0.95$. This construction is motivated by non-coherent construction proposed in [1]. Each codeword from $\mathcal{X}_{A}$ corresponds to a digital symbol $S_{A} \in\{0,1,2,3\}$. By analogy, each codeword from $\mathcal{X}_{B}$ corresponds to a digital symbol $s_{B} \in\{0,1,2,3\}$ We recall that in the non-coherent setup, the subspaces are the informationbearing objects. By transmitting one of the codewords from each set, we transmit 2 bits of information. For each codebook the codewords represent distinct 1-dimensional subspaces from the 4 dimensional (in general) complex space $\mathbb{C}^{4}$, thus being elements (points) from the Grassmann manifold $G_{4,1}^{\mathbb{C}}$. We recall that in the non-coherent setup, the subspaces are the information-bearing objects. The signal received by the relay is

$$
\mathbf{y}_{R}=\sqrt{P T}\left[\begin{array}{ll}
\mathbf{x}_{A} & \mathbf{x}_{B}
\end{array}\right]\left[\begin{array}{l}
h_{A R} \\
h_{B R}
\end{array}\right]+\mathbf{z}_{R},
$$

We denote the set of all matrices obtained by concatenation of $\mathbf{x}_{A}$ and $\mathbf{x}_{B}$ as $\mathcal{Q}=\left\{\mathbf{Q}_{(i, j)}\right\}$, where $\mathbf{Q}_{i, j}$ corresponds to the pair of symbols $\left(S_{A}, S_{B}\right)=(i, j)$. Further, we observe that the columns of the matrix $\mathbf{Q}_{(i, j)}$ span a 2-dimensional subspace, $\Omega_{Q_{i, j}}$. The number of different 2-dimensional subspaces obtained in this way is at most $\left|\mathcal{X}_{A}\right| \cdot\left|\mathcal{X}_{B}\right|$. When designing the codebooks $\mathcal{X}_{A}$ and $\mathcal{X}_{B}$ it is desirable that the codebook $\mathcal{Q}$ obtained by the concatenation of the codewords of both codebooks has certain properties in terms of the design criteria for non-coherent space-time codes, the chordal distance and the diversity product [10] being the most important ones. Here we concentrate on the chordal distance which for two $M$ dimensional subspaces of $\mathbb{C}^{T}, \boldsymbol{\Phi}$ and $\boldsymbol{\Psi}$ is given by

$$
\mathrm{d}_{c}(\langle\boldsymbol{\Phi}\rangle,\langle\boldsymbol{\Psi}\rangle)=\sqrt{\sum_{i=1}^{M} \sin ^{2} \theta_{i}} .
$$

Here $\theta_{i}$ are the principle angles between the subspaces, defined as $\theta_{i}=\arccos \sigma_{i}$, where $\sigma_{i}$ are the singular values in the singular value decomposition (SVD), $\boldsymbol{\Phi}^{\prime} \boldsymbol{\Psi}=\mathbf{U} \boldsymbol{\Sigma} \mathbf{V}^{\mathrm{H}}$. In order to illustrate the properties of the codebook $\mathcal{Q}$, we give it's distance profile, as shown in Fig. 2

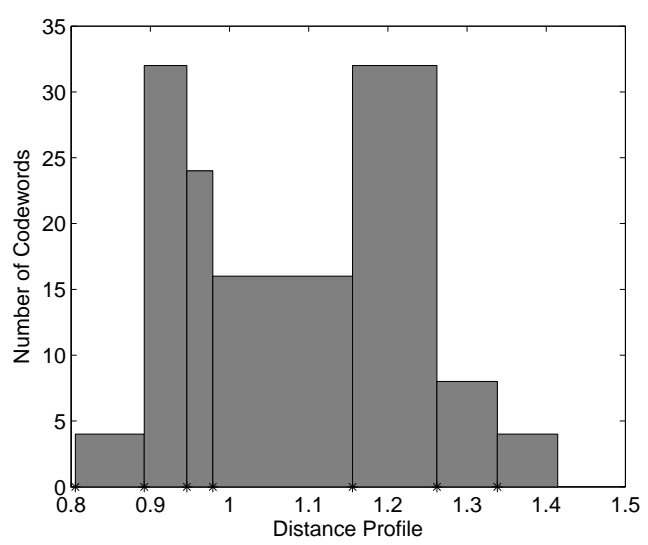

Fig. 1. Distance profile of the codebook $\mathcal{Q}$

Now, based on the received vector $\mathbf{y}_{R}$, i.e. on the subspace the relay has to find a denoising map $\mathcal{C}$. In the following we describe the denoising process in detail.

2) Denoising Map: In terms of the transmit symbols of each user, it turns out that a suitable denoising map would be the following

$\mathcal{C}\left(S_{A}, S_{B}\right)= \begin{cases}0, & \left(S_{A}, S_{B}\right) \in\{(0,0),(1,1),(2,2),(3,3)\} \\ 1, & \left(S_{A}, S_{B}\right) \in\{(0,1),(1,0),(2,3),(3,2)\} \\ 2, & \left(S_{A}, S_{B}\right) \in\{(0,2),(2,0),(1,3),(3,1)\} \\ 3, & \left(S_{A}, S_{B}\right) \in\{(0,3),(3,0),(1,2),(2,1)\}\end{cases}$

It is clear that this map, together with the side information each user has, allows for unambiguous decision at the receive side. What remains is to provide a mechanism for the relay to be able to map the received signal $\mathbf{y}_{R}$ into the symbol $S_{R}=\mathcal{C}\left(S_{A}, S_{B}\right)$. A straight-forward way would be that the relay first makes a ML decision about the 2-dimensional subspace spanned by $\left[\begin{array}{ll}\mathbf{x}_{A} & \mathbf{x}_{B}\end{array}\right]$. The ML decision consists of projection of the received subspace on all possible subspaces and decision about the most probable one based on the Frobenius norm

$$
\mathbf{Q}=\arg \max _{\mathbf{Q}_{i, j} \in \mathcal{Q}}\left\|\mathbf{Y}_{R}^{\mathrm{H}} \mathbf{Q}_{i, j}\right\|_{F}^{2}
$$

The ML decision coincides with the search for the subspace which is at the smallest chordal distance from the received subspace, which justifies the choice of the chordal distance as one of the design criteria. This, together with the map (11), gives the symbol $S_{R}$. We note that because of the 
grouping, the error probability of the relay is smaller than the error probability in the case of decode-and-forward, since it is enough to classify the received subspace in one of the subsets corresponding to the symbols $0,1,2$ and 3 . The probability that the signal received at the relay is correctly mapped to the symbol $S_{R}$ depends on two factors. The first one is the distance profile of the set of subspaces $\mathcal{Q}$ which was previously discussed. The second one is the distribution of the distances between the codewords within one group, i.e. the codewords which are mapped to the same symbol $S_{R}$ and, more important, the distances between codewords from different groups. Let us take one codeword for illustration, for example the codeword $\mathbf{Q}_{0,1}$. According to the denoising map this codeword belongs to the group 1 corresponding to the symbol $S_{R}=1$, together with the codewords $\mathbf{Q}_{1,0}, \mathbf{Q}_{2,3}$ and $\mathbf{Q}_{3,2}$. An error occurs when the symbol $\mathbf{Q}_{0,1}$ is mapped to a symbol from another group. Indeed, if the symbol $\mathbf{Q}_{0,1}$ would have been mistaken for another symbol from the same group, let us say $\mathbf{Q}_{2,3}$, this will not result in error since the symbol $S_{R}=1$ is is still correctly assigned. Based on it's side information, the receiver will be able to deliver the correct decision. Therefore it is desired that codewords with small mutual distances are grouped together as much as possible. However, the grouping should be such that the decision at the terminals is still possible, condition given by the exclusive law [4]. As a simple example, the codewords $\mathbf{Q}_{0,1}$ and $\mathbf{Q}_{0,2}$ can not be grouped together since the user A, for example, based on the symbol it transmitted ( 0 in this case) can not decide about the symbol from user $B$, which can be both 1 and 2 . The most critical factors which dominate the error are the minimal distance between any two codewords from different groups $i$ and $j$, which we denote as $d_{\min }(i, j)$ and the number of codewords at this distance. In the described example we have the following distance matrix between the different groups

$$
D_{\text {min }}=\left[\begin{array}{cccc}
0.8054 & 0.8924 & 0.8054 & 0.8924 \\
0.8924 & 0.9463 & 0.8924 & 0.8054 \\
0.8054 & 0.8924 & 0.8054 & 0.8924 \\
0.8924 & 0.8054 & 0.8924 & 0.9463
\end{array}\right] \text {. }
$$

The events which dominate the error performance are when the symbol $S_{R}=0$ is mistaken with the symbol $S_{R}=2$ and when the symbol $S_{R}=1$ is mistaken with the symbol $S_{R}=3$, and vice versa. There is place for optimization of the chosen constellations in order to decrease the probabilities of this events. The nature of the problem is combinatorial and requires the use of combinatorial methods, as well as some heuristics. This is a topic of current work.

After the grouping, the denoising map $\mathcal{C}$ generates a networkcoded data $S_{R}$ from the ML estimates; i.e., $S_{R}=$ $\mathcal{C}\left(\hat{S}_{A}, \hat{S}_{B}\right)$. The denoised signal is given as $\mathbf{x}_{R}=\mathcal{M}_{R}\left(S_{R}\right)$ and is the signal which is going to be transmitted in the broadcast (BC) stage.

\section{Broadcast (BC) Stage}

In the $\mathrm{BC}$ stage the denoised signal is broadcasted to A and B. Since no channel knowledge is assumed at the terminals $\mathrm{A}$ and $\mathrm{B}$, in this stage we will also use subspacebased communication. The relay codebook should consist of 4 codewords. The choice of the codewords should be based on the criteria for construction of non-coherent codes, the most important being the chordal distance and the diversity product [10]. Previous works on non-coherent space-time coding [8] show that a suitable choice is the following

$$
\mathbf{x}_{R}=\left[\begin{array}{c}
\cos \alpha \\
\sin \alpha r
\end{array}\right]
$$

where $r$ is one of the QPSK symbols. The parameter $\alpha$ should be chosen such that both the minimum diversity sum (chordal distance) and the minimal diversity product in the constellation distance profile are maximized. A possible choice of the codebook $\mathcal{X}_{R}$ is the following

$$
\mathcal{X}_{R}=\left\{\left[\begin{array}{c}
\frac{1}{\sqrt{2}} \\
\frac{1+i}{2}
\end{array}\right],\left[\begin{array}{c}
\frac{1}{\sqrt{2}} \\
\frac{1-i}{2}
\end{array}\right],\left[\begin{array}{c}
\frac{1}{\sqrt{2}} \\
\frac{-1+i}{2}
\end{array}\right],\left[\begin{array}{c}
\frac{1}{\sqrt{2}} \\
\frac{-1-i}{2}
\end{array}\right]\right\}
$$

We note that in the $\mathrm{BC}$ stage it is enough to use $T_{2}$ time slots, where $T_{2}<T$, due to the reduced cardinality after the denoising. In the particular example, two time slots are sufficient to transmit the information beard by one of the 4 subspaces, i.e. $T_{2}=2$. The received signals at the nodes are written as

$$
\mathbf{y}_{A}=\sqrt{P_{R} T_{2}} \mathbf{x}_{R} h_{R A}+\mathbf{z}_{A}
$$

and

$$
\mathbf{y}_{B}=\sqrt{P_{R} T_{2}} \mathbf{x}_{R} h_{R B}+\mathbf{z} .
$$

Now, both terminal A and terminal B can detect the desired data $S_{B}$ and $S_{A}$ respectively, by using the own information $S_{A}$ and $S_{B}$.

\section{SCENARIO 2: RELAY Without CHANNEL} KNOWLEDGE, USERS WITH CSIR OF THE LINK RELAY-USER

In this case we assume that the relay has no channel knowledge and the users have channel knowledge of the link relay-user. This case is different from the case when the users have CSIR of the links terminal-relay and relay-terminal. In a certain way, this assumption is more realistic, since if the relay $\mathrm{R}$ decodes the signal first, or performs denoise-and-forward, it is not easy for the user B to learn about the channel between user $\mathrm{A}$ and the relay $\mathrm{R}$. It might be more realistic that the user $\mathrm{B}$ has a knowledge about the product channel A-R-B (if this information is sent in an AF fashion), or only of the link R-B, if the relay re-sends the pilot symbols.

A possible scenario is described as follows. The users A and B use non-coherent codebooks (sets of subspaces) and the relay performs denoise-and-forward, as described in the previous section. The constellation mapper at the relay uses a coherent modulation scheme since the terminals have CSIR. The users then decode based on the symbol they detect, their own transmit symbol and the channel knowledge about the link relay-user. As example we use the same codebooks $\mathcal{X}_{A}$ and $\mathcal{X}_{B}$ and the same denoising map as in the non-coherent case. After the received signal at the relay $x_{R}$ is mapped to the relay symbol $S_{R}$, we use a simple QPSK constellation mapper 
$\mathcal{M}\left(S_{R}\right)$ for the signal in the broadcast stage. The MA stage requires four time slots and the $\mathrm{BC}$ stage one time slot.

A comparison with an AF scheme for the same scenario is not performed here, since an AF scheme can not benefit from the receive channel knowledge of the channel relay-terminal only. However, if we assume that the terminals can obtain an estimate of the product channel terminal-relay-terminal, then an AF scheme would benefit from this channel knowledge.

\section{Simulation RESUlts}

\section{A. Scenario 1}

We present a comparison between the proposed DNF scheme and a scheme based on AF. For both schemes we use the codebooks $\mathcal{X}_{A}$ and $\mathcal{X}_{B}$ as defined in Section III. In the DNF scheme we map the denoised symbols to subspaces from an alphabet adapted to the links relay-terminal. Since the cardinality of this codebook is reduced (in this case 4), it suffices to send the information in 2 time slots, compared to the 4 time slots needed for the AF scheme, where the signal received at the relay is only scaled and retransmitted. The particular choice of the alphabets, as given in Section III, results in an effective rate (in total) of $\eta=0.66$ b.c.u for the DNF scheme and $\eta=0.5$ b.c.u for the AF scheme. The simulation results are presented in Fig. 1. We see that the DNF scheme outperforms the AF scheme in most of the SNR region, and this at a higher effective rate, due to the time slots saved in the BC stage.

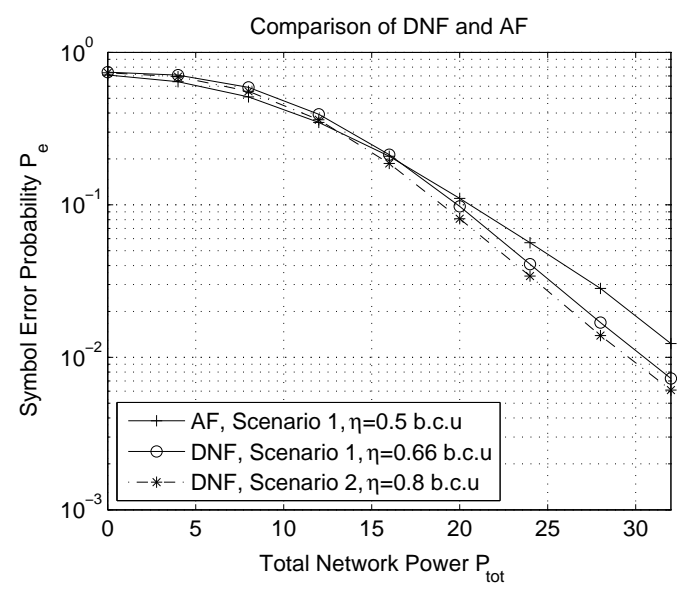

Fig. 2. Performance comparison of DNF and AF

\section{B. Scenario 2}

Here we compare the performance of the DNF scheme in the second scenario with the performance of the same scheme in the first scenario. For both schemes we use the codebooks $\mathcal{X}_{A}$ and $\mathcal{X}_{B}$ as defined in Section III. In the BC stage we map the denoised symbols to symbols from a QPSK constellation. It suffices to send the information in the BC stage in one time slot which results in an effective rate (in total) of $\eta=0.8$ b.c.u. The simulation results are presented in Fig. 1. The performance of the DNF scheme in this scenario is slightly better than in the first scenario, at a higher rate. However, the performance is dominated by non-coherent part of the transmission, i.e. the MA stage, which is expected. The receive channel knowledge of the link relay-terminal does not improve the end-to-end performance dramatically, which is expected.

\section{CONCLUSIONS AND FUTURE WORK}

We investigated non-coherent and semi-coherent schemes for physical-layer network coding in two-way relaying scenarios. We distinguished between two scenarios. In the first scenario neither the relay nor the terminals had channel knowledge, termed as non-coherent communication. In the second scenario we assumed that the terminals have receive channel knowledge of the link relay-terminal (semi-coherent communication). We combined the paradigm of subspacebased communication originally developed for non-coherent point-to-point channels, with two-way relaying schemes based on physical-layer wireless network coding with denoise-andforward (DNF). The performance comparison shows improvement over a scheme based on amplify-and-forward (AF). The results can be further extended to more general constellation designs as well to the search of constellation which are optimal in the DNF setup. Additionally, other scenarios can be addressed, for example the scenario which involves receive channel knowledge at the relay and no channel knowledge at the terminals. This might be relevant when the relay plays the role of a base station, i.e. acts as an infrastructure relay.

\section{REFERENCES}

[1] T. L. Marzetta and B. M. Hochwald, "Capacity of a mobile multiple antenna communication link in Rayleigh flat fading," IEEE Transactions on Information Theory, vol. 45, no. 1, pp. 139-157, January 1999.

[2] Z. Utkovski, A. Sezgin and J. Lindner, "Non-Coherent Two-Way Relaying: Rate Bounds for the high SNR Regime," In Proc. IEEE International Symposium on Information Theory Applications, ISITA 2010, Taichung, October 2010.

[3] Z. Utkovski, Y. Cheng and J. Lindner, "Non-Coherent AF Scheme for Two-Way Wireless Relay Networks based on Packings in Grassmann Manifolds," In Proc. IEEE International Symposium on Information Theory Applications, ISITA 2010, Taichung, October 2010.

[4] P. Popovski, H. Yomo "Wireless Network Coding by Amplify-andForward for Bi-Directional Traffic Flows," IEEE Communications Letters, vol. 11, no 1, Jan. 2007.

[5] L. Zheng and D. Tse, "Communication on the Grassmann manifold: a geometric approach to the non-coherent multiple antenna channel," IEEE Trans. Inform. Theory, vol. 46, pp. 1456-1467, 1999.

[6] P. Popovski and $\mathrm{H}$. Yomo, Bi-directional amplification of throughput in a wireless multi-hop network, IEEE VTC2006, Melbourne, May 2006.

[7] T. Koike-Akino, P. Popovski and V. Tarokh," Denoising Maps and Constellations for Wireless Network Coding in Two-Way Relaying Systems," Globecom 2008, December 2008.

[8] Z. Utkovski, G. Yammine and J. Lindner, "A Differential Scheme for Two-Way Relaying Channels", In Proc. ISIT 2009, Seoul, June 2009.

[9] N. J. A. Sloane, Packing planes in four dimensions and other mysteries, Algebraic Combinatorics and Related Topics, 1997.

[10] O. Henkel, "Geometrical relations between space time block code designs and complexity reduction, "IEEE Trans. Inform. Theory, vol. 52, no. 12 , pp. 5324-5335, Dec. 2006.

[11] J. A. Tropp, I. S. Dhillon, R. W. Heath Jr., T. Strohmer "Designing structured tight frames via an alternating projection method", IEEE Trans. Inform. Theory, vol. 51, no. 1, January 2005. 